\title{
Nox4 Is a Major Source of Superoxide Production in Human Brain Pericytes
}

\author{
Junya Kuroda ${ }^{a}$ Tetsuro Ago $^{a}$ Ataru Nishimura ${ }^{a}$ b Kuniyuki Nakamura ${ }^{a}$ \\ Ryu Matsuo ${ }^{a}$ Yoshinobu Wakisaka ${ }^{a}$ Masahiro Kamouchi ${ }^{a}$ Takanari Kitazono ${ }^{a}$ \\ Departments of a Medicine and Clinical Science and ${ }^{b}$ Neurosurgery, Graduate School of Medical Sciences, \\ Kyushu University, Fukuoka, Japan
}

\section{Key Words}

Pericytes $\cdot$ NADPH oxidase $\cdot$ Nox4 $\cdot$ Reactive oxygen species · Proliferation · Angiogenesis · Angiotensin II · Hypoxia $\cdot$ Brain ischemia

\begin{abstract}
Background: Pericytes are multifunctional cells surrounding capillaries and postcapillary venules. In brain microvasculature, pericytes play a pivotal role under physiological and pathological conditions by producing reactive oxygen species (ROS). The aims of this study were to elucidate the source of ROS and its regulation in human brain pericytes. Methods: The expression of Nox enzymes in the cells was evaluated using RT-PCR and western blot. Superoxide production was determined by superoxide dismutase-inhibitable chemiluminescence. Silencing of Nox4 was performed using RNAi, and cell proliferation was evaluated using the 3-(4,5-dimethylthiazol-2-yl)-5-(3-carboxymethoxyphenyl)-2-(4sulfophenyl)-2H-tetrazolium (MTS) assay. Results: Nox4 was predominant among the Nox family in human brain pericytes. Membrane fractions of cells produced superoxide in the presence of $\mathrm{NAD}(\mathrm{P}) \mathrm{H}$. Superoxide production was almost abolished with diphenileneiodonium, a Nox inhibitor; however, inhibitors of other possible superoxide-producing enzymes had no effect on $\mathrm{NAD}(\mathrm{P}) \mathrm{H}$-dependent superoxide
\end{abstract}

production. Pericytes expressed angiotensin II (Ang II) receptors, and Ang II upregulated Nox4 expression. Hypoxic conditions also increased the Nox4 expression. Silencing of Nox4 significantly reduced ROS production and attenuated cell proliferation. Conclusion: Our study showed that Nox4 is a major superoxide-producing enzyme and that its expression is regulated by Ang II and hypoxic stress in human brain pericytes. In addition, Nox4 may promote cell growth.

() 2015 S. Karger AG, Basel

\section{Introduction}

Pericytes are multifunctional cells surrounding microvessels, such as capillaries and postcapillary venules [1], and interact closely with vascular endothelial cells, modulate endothelial function and regulate microvascular blood flow $[2,3]$. Although pericytes exist on microvessels in all organs, the density of pericytes varies according to the function of the vessel and organ in which the cells reside. The highest density of pericytes in the body is found in vessels of neural tissues, such as the brain and retina $[1,4]$. In cerebral vessels, pericytes are proposed to have important roles in the regulation of cerebral microcirculation and angiogenesis, maintenance of the blood-brain barrier, and immune response [5-9].

\section{KARGER}

(C) 2015 S. Karger AG, Base

$1018-1172 / 15 / 0516-0429 \$ 39.50 / 0$

E-Mail karger@karger.com

www.karger.com/jvr
Dr. Junya Kuroda

Department of Medicine and Clinical Science

Graduate School of Medical Sciences, Kyushu University

3-1-1 Maidashi, Higashi-ku, Fukuoka 812-8582 (Japan)

E-Mail j-kuro@intmed2.med.kyushu-u.ac.jp 
In vascular walls, reactive oxygen species (ROS) appear to play a pivotal role in a variety of pathophysiological processes, such as the development of arteriosclerosis and disturbance of vessel tone regulation [10-13]. Recent evidence has suggested that the major source of ROS in the vascular wall is NADPH oxidase (Nox) [14-16]. The Nox family enzymes are dedicated to ROS production. The founder member of the Nox family is gp91phox, a membrane-spanning protein, which is predominantly expressed in professional phagocytes and has a crucial role in killing microorganisms [17]. p22 phox, another membrane-integrated protein, forms a heterodimer with gp91 ${ }^{\text {phox }}$, thereby stabilizing gp91 ${ }^{\text {phox }}$ [17-19]. ROS are also produced in non-phagocytic cells, such as vascular cells. Seven members of the Nox family have been identified so far (Nox1-5 and Duox 1 and 2; gp91 ${ }^{\text {phox }}$ was renamed as Nox2). Nox family members including Nox1, Nox3, Nox4, and p22 phox also form heterodimers, stabilizing each other [17-19]. We have shown previously that Nox 4 is expressed abundantly in the vascular smooth muscle and endothelial cells [20-22]. However, the expression of Nox enzymes in pericytes is still unknown. Thus, the aims of the present study were to investigate the expression of Nox enzymes, monitor the production of ROS, and identify the functional role and regulation of Nox enzymes in brain microvascular pericytes.

\section{Methods}

\section{Cell Culture}

Human brain microvascular pericytes were purchased from the Applied Cell Biology Research Institute and cultured in CS-C complete medium (Cell Systems Corp., Kirkland, Wash., USA) containing $10 \%(\mathrm{v} / \mathrm{v})$ fetal bovine serum. The manufacturer confirmed that the cells scarcely exhibited cytoplasmic uptake of Dil-Ac-LDL, inducible expression of E-Selectin (CD 62E), and expression of von Willebrand Factor (vWF), and did express vimentin intermediate filaments. Cells of passage 4-10 were used for experiments.

\section{RT-PCR}

Total RNA of indicated cells were prepared using TRIZOL reagent (Life Technologies Inc., Carlsbad, Calif., USA). Total RNA were subjected to reverse-transcription polymerase chain reaction (RT-PCR) using the ReverTra Ace qPCR RT kit (TOYOBO, Osaka, Japan) with pairs of specific primers: $5^{\prime}$-CACTGCCTG TCCCCTATGAT- $3^{\prime}$ (forward primer) and $5^{\prime}$-ACAGTCTGCA CTGCGTTCAC-3' (reverse primer) for platelet-derived growth factor receptor- $\beta$ (PDGFR- $\beta$ ); $5^{\prime}$-ACACAAGTGTCTGGCTGA GG-3' (forward primer) and $5^{\prime}$-CAACAGAGCCATTGGTGCA G-3' (reverse primer) for vWF; 5'-CTGGAGGTTGAGAGGGA CAA- $3^{\prime}$ (forward primer) and $5^{\prime}$-CTCCAGCGACTCAATCTT CC- $3^{\prime}$ (reverse primer) for glial fibrillary acidic protein (GFAP); 5'-GCAAGATCTGTTGTTATGCACCCATCCAA-3' (forward primer) and $5^{\prime}$-GCTGGTACCTCAAAAATTTTCTTTGTTGAA GT-3' (reverse primer) for Nox1; 5'-GGAGGATCCGTGGTCA CTCACCCTTTCAA- $3^{\prime}$ (forward primer) and $5^{\prime}$-CCACTCGAG CTCATGGAAGAGACAAGTTAG-3' (reverse primer) for Nox2; 5'-GCAGGATCCGTGGTAAGCCACCCCTCTG-3' (forward primer) and $5^{\prime}$-GCTGAATTCAGAAGCTCTCCTTGTTGTAA T-3' (reverse primer) for Nox3; 5'-GCAGGATCCGTCATAAGT CATCCCTCAGA-3' (forward primer) and $5^{\prime}$-GCTGTTAAC GTCGACTCAGCTGAAAGACTCTTTAT-3' (reverse primer) for Nox4; 5'-GCAGGATCCACTATCTGGCTGCACATTCG-3' (forward primer) and $5^{\prime}$-GCTGAATTCCTAGAAATTCTCTTG GAAAAATC-3' (reverse primer) for Nox5; 5'-AGTGTGTTTC TACTCACGTG- $3^{\prime}$ (forward primer) and $5^{\prime}$-TTATCTGAGGG GCGGTAGGA-3' (reverse primer) for angiotensin type-1 receptor (AT1R); $5^{\prime}$-TGAGTGTTGATAGGTACCAA-3' (forward primer) and $5^{\prime}$-CCATTTCTCTAAGAGAACTG- $3^{\prime}$ for angiotensin type-2 receptor (AT2R); 5'-CAAGAGATGGCCACGGCT GCT- $3^{\prime}$ and $5^{\prime}$-TCCTTCTGCATCCTGTCGGCA- $3^{\prime}$ for $\beta$-actin.

Briefly, $1 \mu \mathrm{l}$ of the reverse transcription reaction was used for PCR amplification in a volume of $50 \mu$ containing the gene specific primers described above. PCR was performed using TaKaRa Taq Hot Start Version at $94^{\circ} \mathrm{C}$ for $5 \mathrm{~min}$, followed by 30 cycles at $94^{\circ} \mathrm{C}$ for $30 \mathrm{~s}, 57^{\circ} \mathrm{C}$ for $30 \mathrm{~s}, 72^{\circ} \mathrm{C}$ for $1 \mathrm{~min}$, and finally $72^{\circ} \mathrm{C}$ for $7 \mathrm{~min}$. PCR products were visualized on a $1.2 \%(\mathrm{w} / \mathrm{v})$ agarose gel stained with ethidium bromide.

\section{Middle Cerebral Artery Occlusion Model and}

\section{Immunofluorescence}

We used a male FVB mouse aged 10 weeks. Focal brain ischemia was produced using permanent occlusion of the right middle cerebral artery (MCAO) as described previously $[23,24]$. The operated mouse was sacrificed with deep anesthesia 4 days after MCAO and was perfused transcardially with ice-cold heparinized saline and $4 \%(\mathrm{w} / \mathrm{v})$ paraformaldehyde. The brain was quickly removed and fresh coronal sections ( $2 \mu \mathrm{m}$ thick) were fixed with $4 \%$ (w/v) paraformaldehyde in phosphate-buffered saline (PBS) for immunofluorescence. Paraformaldehyde-fixed coronal sections were embedded in the optimum cutting temperature compound and were cut into $20 \mu \mathrm{m}$ thick slices. Sections were blocked with PBS containing $5 \%$ normal goat serum for $1 \mathrm{~h}$ at room temperature. Next, the sections were incubated with primary antibodies against Nox4 (Abcam, Cambridge, UK) and PDGFR- $\beta$ (Cell Signaling Technology, Danvers, Mass., USA) overnight at $4^{\circ} \mathrm{C}$. Secondary antibody (affinity-purified anti-rabbit IgG) was incubated for $30 \mathrm{~min}$ at room temperature. The slides were observed using Z-stack function ( $2 \mu \mathrm{m}$ intervals) on a BIOREVO BZ-9000 fluorescence microscope (Keyence, Osaka, Japan). The animal experiments were approved by the animal ethics committee of Kyushu University.

Preparation of Membrane and Cytosolic Fractions of Human Brain Pericytes

The membrane fraction enriched in integral membrane and membrane-associated proteins and the cytosol fraction of human brain pericytes were prepared using the ProteoExtract Native Membrane Protein Extraction Kit (Merck Millipore, Billerica, Mass., USA) according to the manufacturer's protocol. Briefly, the cells were washed with ice-cold wash buffer, and subjected to Extraction Buffer I with Protease Inhibitor Cocktail. After incubation on ice for 10 min under gentle agitation, the resultant supernatant was transferred into a sample tube and 
used as the cytosolic fraction. The residual attached on the plate was further subjected to Extraction Buffer II with Protease Inhibitor Cocktail. After incubation on ice for $30 \mathrm{~min}$, the supernatant was transferred into a sample tube and used as the membrane fraction.

\section{Assay for Superoxide Production Using Chemiluminescence}

Superoxide production was determined as superoxide dismutase (SOD)-inhibitable chemiluminescence detected with an enhancer-containing luminol-based detection system (Diogenes; National Diagnostics, Atlanta, Ga., USA) as described previously $[21,25]$. Briefly, the membrane and the cytosol fractions of human brain pericytes ( $5 \mu \mathrm{g}$ protein) were suspended in $200 \mu \mathrm{l}$ of an assay buffer composed of $100 \mathrm{~mm}$ potassium phosphate $(\mathrm{pH}$ 7.0), $10 \mu \mathrm{M} \mathrm{FAD}, 1 \mathrm{mM} \mathrm{NaN}_{3}$, and $1 \mathrm{mM}$ EGTA. After preincubation with the enhanced luminol-based substrate $(200 \mu \mathrm{l}), \mathrm{NADH}$ or NADPH was added at a final concentration of $500 \mu \mathrm{M}$. The chemiluminescence was monitored continuously using a luminometer (MiniLumat LB9506; EG\&G Berthold). The reaction was terminated by the addition of SOD $(100 \mu \mathrm{g} / \mathrm{ml})$.

\section{Assay for Hydrogen Peroxide $\left(\mathrm{H}_{2} \mathrm{O}_{2}\right)$ Production}

$\mathrm{H}_{2} \mathrm{O}_{2}$ production was measured with an Amplex Red $\mathrm{H}_{2} \mathrm{O}_{2}$ assay kit (Molecular Probes, Eugene, Oreg., USA) according to the manufacturer's instructions as described previously [26]. Briefly, the membrane fraction $(5 \mu \mathrm{g})$ was suspended with a working solution containing Amplex Red $(100 \mu \mathrm{M})$, horseradish peroxidase $(0.2$ $\mathrm{U} / \mathrm{ml}$ ), and $500 \mu \mathrm{M} \mathrm{NADH}$. After incubation for $30 \mathrm{~min}$ at room temperature, the absorbance was measured $(540 \mathrm{~nm})$ using a microplate reader.

\section{RNAi for Nox4 Knockdown}

RNAi was performed according to the method by Elbashir et al. [27]. The double-strand siRNAs targeting Nox4 were prepared as a $3^{\prime}$-overhang form using sense and anti-sense oligoribonucleotides. The sequences were $5^{\prime}$-CCUUCUUGCUGUAUAACC ATT-3' (sense) and 5'-UGGUUAUACAGCAAGAAGGTT-3' (anti-sense) for Nox4. Silencer Negative Control \#1 siRNA (Life Technologies) was used as a negative control. For transfection of human brain pericytes with siRNA, we used Nucleofector technology (Lonza, Basel, Switzerland), an electroporation-based gene transfer technique, according to the manufacturer's protocol. Briefly, human brain pericytes were suspended in the Nucleofector Solution at a final concentration of $0.5-1 \times 10^{6} \mathrm{cells} / 100 \mu \mathrm{l}$, and $1 \mu \mathrm{g}$ of siRNA duplex was added to the sample. The sample was transferred into a certified cuvette, and subjected to the Nucleofector, which was programmed for optimized transfection of pericytes. After transfection, the cells were cultured for $48 \mathrm{~h}$ or longer in the medium described above, and used for the following experiments.

\section{Quantitative Real-Time PCR}

mRNA expression of Nox 4 and p22 $2^{\text {phox }}$ was quantified by realtime PCR of reverse-transcribed cDNA of these cells using the LightCycler instrument (Roche, Basel, Switzerland). Primer sequences for Nox4 and p22 phox were as follows: $5^{\prime}$-CTTCCGTTG GTTTGCAGATT-3' (forward primer) and $5^{\prime}$-TGGGTCCACAA CAGAAAACA-3 ${ }^{\prime}$ (reverse primer) for Nox $4 ; 5^{\prime}$-GTACTTTGGT GCCTACTCCA- $3^{\prime}$ (forward primer) and $5^{\prime}$-CGGCCCGAA CATAGTAATTC-3' (reverse primer) for $\mathrm{p} 22^{\text {phox }}$.
Western Blotting

Cultured cells were washed twice in PBS and homogenized in the radioimmunoprecipitation assay (RIPA) lysis buffer (50 $\mathrm{mm}$ Tris-HCl, pH 7.5, $150 \mathrm{~mm} \mathrm{NaCl}, 1 \%$ (v/v) NP-40, 0.5\% (w/v) deoxycholic acid, $0.1 \%$ (w/v) SDS, $5 \mathrm{~mm}$ EDTA, $10 \mathrm{mM} \mathrm{Na}_{4} \mathrm{P}_{2} \mathrm{O}_{7}$, $0.1 \mathrm{mM} \mathrm{Na} \mathrm{VO}_{4}, 1 \mathrm{mM} \mathrm{NaF}$, and protease inhibitor cocktail (Thermo Scientific, Rockford, Ill., USA)). Protein concentration was determined using the BCA Protein Assay Kit (Thermo Scientific). Samples were subjected to SDS-polyacrylamide gel electrophoresis $(20 \mu \mathrm{g} / \mathrm{lane})$ and then transferred onto polyvinylidene fluoride membranes. The membranes were incubated for $1 \mathrm{~h}$ with ECL-prime blocking reagent (GE Healthcare, Piscataway, N.J., USA) at room temperature, and probed overnight at $4{ }^{\circ} \mathrm{C}$ with the primary antibodies against Nox4 (Abcam, Cambridge, UK) or $\beta$-actin (Sigma-Aldrich, St. Louis, Mo., USA). Membranes were then washed and incubated with secondary antibodies (1:100,000 dilution, Cell Signaling Technology, Danvers, Mass., USA) for $30 \mathrm{~min}$ at room temperature. Blots were developed using Immunostar LD (WAKO, Osaka, Japan) according to the manufacturer's instructions. Quantification of each band was performed using Image J software (NIH).

\section{Assay for Cell Proliferation}

We performed a cell proliferation assay using the CellTiter $96^{\circledR}$ Aqueous One Solution Cell Proliferation Assay Kit (Promega, Fitchburg, Wisc., USA) containing 3-(4,5-dimethylthiazol-2-yl)5-(3-carboxymethoxyphenyl)-2-(4-sulfophenyl)-2H-tetrazolium, inner salt (MTS), according to the manufacturer's instructions. Briefly, human brain pericytes were subjected to transfection with siRNA. Thereafter, the cells were seeded onto a collagen-coated 96-well plate. Forty-eight or $120 \mathrm{~h}$ later, $20 \mu \mathrm{l}$ of the reagent was added into each well of the 96-well assay plate containing $100 \mu \mathrm{l}$ of culture medium. The plate was incubated for $1 \mathrm{~h}$ in a humidified, $5 \%(\mathrm{v} / \mathrm{v}) \mathrm{CO}_{2}$ atmosphere, and then the absorbance at $490 \mathrm{~nm}$ was recorded using 96-well plate reader.

\section{Statistical Analysis}

All values are expressed as the mean \pm SD. Statistical analyses between groups were performed by the unpaired Student t-test or one-way analysis of variance followed by a Bonferroni post hoc test. A value of $\mathrm{p}<0.05$ was considered statistically significant.

\section{Results}

\section{Expression of Nox in Human Brain Pericytes and} Microvessels in a Mouse Brain

To confirm the integrity of the purchased human brain pericytes, we checked the expression of markers for cells constituting capillaries in the brain, namely PDGFR- $\beta$ (for pericytes), vWF (for endothelial cells), and GFAP (for astrocytes), using RT-PCR. We found that only PDGFR- $\beta$ was expressed, indicating that the cells were pericytes (fig. 1a). We investigated the expression of the Nox family members in the human brain pericytes using 


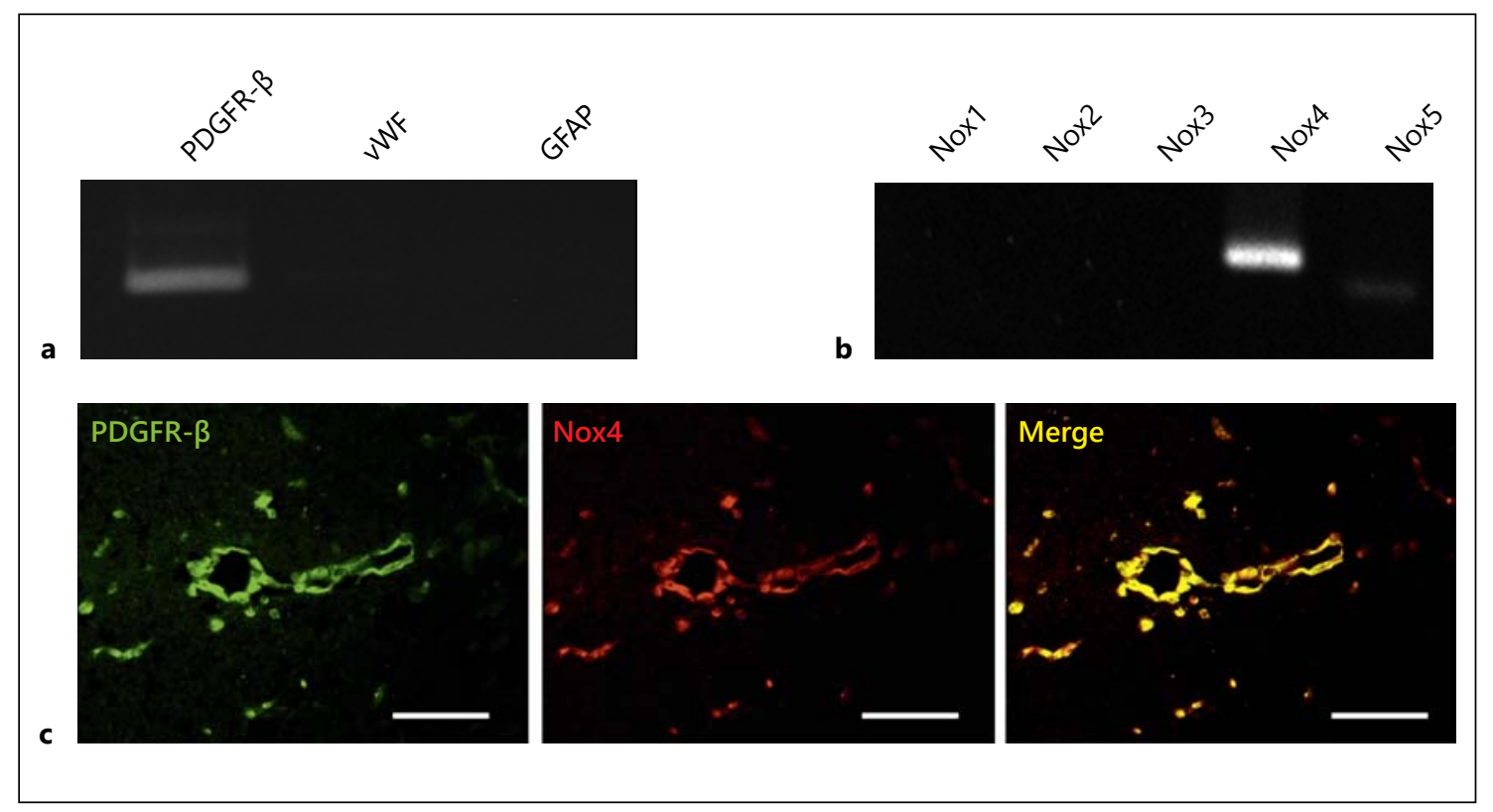

Fig. 1. Expression of Nox family enzymes in human brain pericytes. a Integrity of human brain pericytes was confirmed using RT-PCR. Expression of markers for cells constituting capillaries in the brain, namely PDGFR- $\beta$ (for pericytes), vWF (for endothelial cells), GFAP (for astrocytes). b Expression of Nox family enzymes in human brain pericytes. RT-PCR was performed using RNA

RT-PCR. As shown in fig. 1b, Nox4 was highly expressed and Nox 5 showed faint expression in pericytes, whereas other Nox enzymes were scarcely expressed. Immunofluorescent staining demonstrated that Nox4 was present in the cells expressing PDGFR- $\beta$ in microvessels in the peri-infarct areas of a mouse stroke model (fig. 1c). These results suggest that Nox 4 is expressed not only in cultured brain pericytes but also in pericytes of microvessels in a mouse brain.

\section{Superoxide Production by Human Brain Pericytes}

To investigate the superoxide-producing activity of human brain pericytes, we prepared membrane and cytosolic fractions of pericytes and measured superoxideproducing activity using a chemiluminescence method. As shown in fig. $2 \mathrm{a}$, the membrane fraction produced superoxide in response to the addition of NADH as well as NADPH. The cytosolic fraction also exhibited both $\mathrm{NADH}$ - and NADPH-dependent superoxide producing activity, but to a lesser extent (fig. 2b). To investigate the effects of cytosolic factors on superoxide-producing activity, we added the cytosolic fraction to the membrane fraction and measured the superoxide-producing activity. As shown in fig. $2 c$, the addition of the cytosolic frac-
$(1 \mu \mathrm{g})$ from human brain pericytes with specific primers for the main members of the Nox family. c Immunofluorescent analysis of PDGFR- $\beta$ (green) and Nox4 (red) in peri-infarct area in a mouse brain at day 4 after permanent middle cerebral artery occlusion (scale bars: $40 \mu \mathrm{m}$ ).

tion had no effects. These results were consistent with the fact that cytosolic proteins are not involved in the superoxide-producing activity of Nox4.

\section{Effects of Inhibitors on Superoxide Production by the Membrane Fraction}

To investigate whether Nox 4 is a source of ROS in the brain pericyte membrane, we tested the effects of inhibitors on several potential superoxide-producing enzymes. SOD completely abolished chemiluminescence, indicating that the chemiluminescence signal was produced by superoxide. Superoxide-producing activity of the membrane fraction was significantly attenuated by diphenyleneiodonium (DPI), an inhibitor of Nox enzymes, in a dose-dependent manner (fig. 2d). Because DPI may inhibit other superoxide-producing flavoproteins, such as nitric oxide synthases, xanthine oxidase, and mitochondrial respiratory chain complex I NADH reductase, we tested the effects of inhibitors for these proteins, namely rotenone (a blocking agent of mitochondrial respiratory chain complex I), L-NG nitroarginine methyl ester (LNAME) (an inhibitor of nitric oxide synthases), and oxypurinol (an inhibitor of xanthine oxidase) on $\mathrm{NAD}(\mathrm{P}) \mathrm{H}$ dependent superoxide production. These inhibitors did 


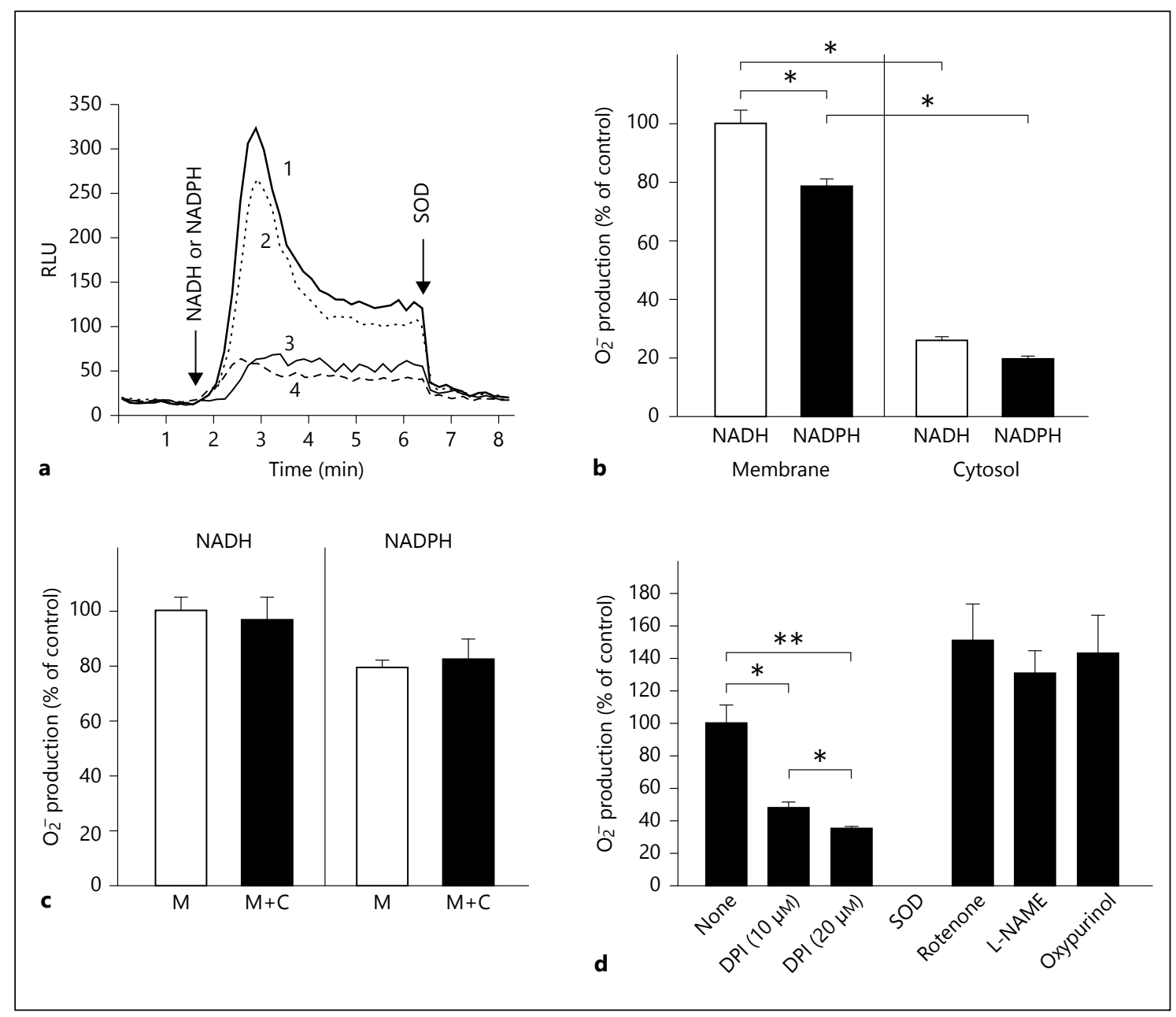

Fig. 2. NADH- or NADPH-dependent superoxide production by human brain pericytes. a NADH- or NADPH-dependent chemiluminescence in the membrane or cytosolic fraction of human brain pericytes. After preincubation of the membrane (traces 1 and 2) or the cytosolic (traces 3 and 4) fraction of human brain pericytes $(5 \mu \mathrm{g})$ for $5 \mathrm{~min}, 500 \mu \mathrm{M} \mathrm{NADH}$ (traces 1 and 3) or NADPH (traces 2 and 4) was added to the reaction mixture and the change in chemiluminescence was continuously monitored with the enhanced luminol-based substrate Diogenes. SOD was added at the final concentration of $50 \mu \mathrm{g} / \mathrm{ml}$ (as indicated by the arrow). b Superoxideproducing activity of the membrane (left panel) or the cytosol (right panel) fraction of human brain pericytes following the addition of
NADH or NADPH $(n=4) .{ }^{*} \mathrm{p}<0.01$. c Effect of the addition of the cytosolic fraction on superoxide-producing activity in the membrane fraction following addition of NADH (left panel) or NADPH (right panel) $(n=4) . M=$ membrane fraction; $M+C=$ membrane plus cytosolic fraction. $\mathbf{d} \mathrm{NADH}$-dependent superoxide production of the membrane fraction in the presence of various inhibitors. The membrane fraction was preincubated for $5 \mathrm{~min}$ in the presence of DPI $(10 \mu \mathrm{M})$, rotenone $(50 \mu \mathrm{M})$, L-NAME $(100 \mu \mathrm{M})$, or oxypurinol (1 $\mathrm{mM})$, followed by the addition of NADH. The SOD-inhibitable chemiluminescence change was continuously monitored with Diogenes. The average value of the activity in the absence of the inhibitors was set as $100 \%(\mathrm{n}=3) .{ }^{*} \mathrm{p}<0.05,{ }^{* *} \mathrm{p}<0.01$. not reduce superoxide-producing activity, suggesting that flavoproteins other than Nox 4 are not involved in the superoxide production of the pericyte membrane (fig. $2 \mathrm{~d}$ ).

\section{Inhibition of Nox4 Expression Reduces ROS \\ Production}

To further confirm that Nox4 is responsible for ROS production in brain pericytes, Nox4 was knocked down using RNAi. We confirmed that RNAi drastically inhibited Nox4 expression at both the mRNA (fig. 3a, b) and protein (fig. 3c, d) level. Upregulation of Nox isoforms other than Nox4 was not found (fig. 3a). The membrane fraction prepared from human brain pericytes transfected with siRNA targeting Nox4 exhibited significantly lower superoxide-producing activity than that of cells transfected with control RNA (fig. 3e). The superoxide 


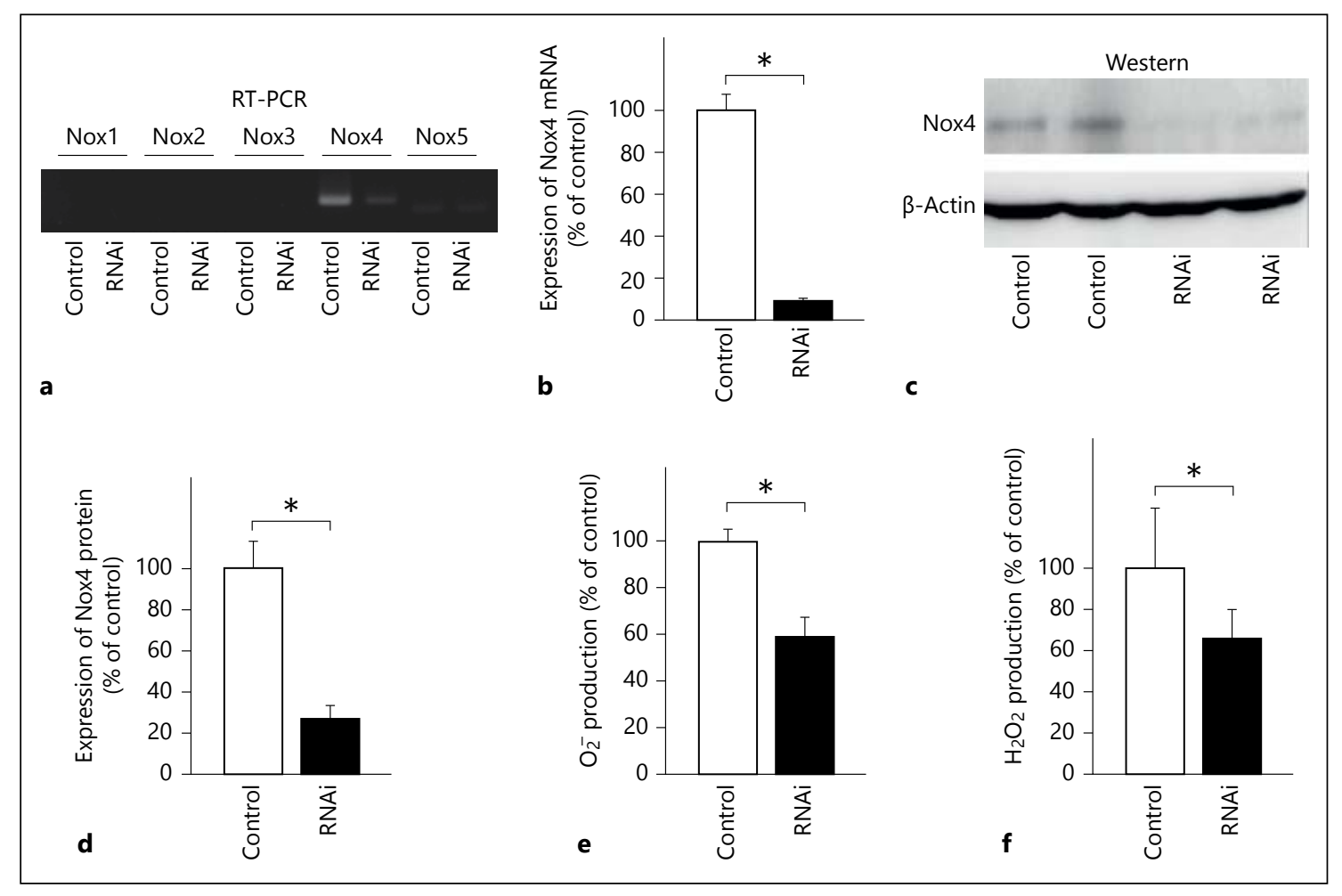

Fig. 3. RNAi-mediated knockdown of Nox4 in human brain pericytes. a, b Effect of Nox 4 siRNA on the expression of Nox 4 mRNA. Human brain pericytes were transfected with 75 pmol of siRNA duplex for Nox 4 by an electroporation-based gene transfer technique (Nucleofector). After culture for $48 \mathrm{~h}$, total RNAs of the transfected cells were prepared and subjected to conventional RTPCR analysis with the primers for Nox isoforms followed by agarose gel electrophoresis (a) and quantitative real-time PCR analysis of Nox4 expression $(n=3)(\mathbf{b})$. c, d Effect of Nox4 siRNA on Nox4 protein expression. Cell lysates from human brain pericytes

production by Nox4 is rapidly converted to $\mathrm{H}_{2} \mathrm{O}_{2}$, which may also be generated directly by Nox4. The Amplex Red assay showed that knockdown of Nox4 significantly inhibited $\mathrm{H}_{2} \mathrm{O}_{2}$ production also (fig. $3 \mathrm{f}$ ). These results suggest that Nox4 is a major source of $\mathrm{NAD}(\mathrm{P}) \mathrm{H}$-dependent ROS production by the membrane fraction of human brain pericytes.

\section{Nox4 Is Upregulated by Angiotensin II (Ang II) and Hypoxic Stress}

We next investigated the regulation of Nox4 expression. The renin-angiotensin system (RAS) is known to work not only as a vasoconstrictor in systemic circulation but also as a mediator of various pathophysiological processes through ROS production by Nox [10]. We found that human brain pericytes expressed both the were subjected to western blotting using an antibody against Nox4. Gel image (c) and its quantitative analysis using imageJ (d) are shown. The average value of the density of bands corresponding to control cells was set as $100 \%(n=4)(\mathbf{d})$. e, $\mathbf{f}$ Effect of Nox4 knockdown on the ROS-producing activity of the membrane fraction of human brain pericytes. Forty-eight $\mathrm{h}$ after transfection of siRNA for Nox4, NADH-dependent production of superoxide (e) and $\mathrm{H}_{2} \mathrm{O}_{2}$ (f) of the membrane fraction was measured. The average value of the activity of control cells was set as $100 \%(n=3)$. $* \mathrm{p}<0.01$.

AT1R and AT2R with the predominance of the AT1R (fig. 4a). Ang II upregulated the expression of Nox4 and p $22^{\text {phox }}$ (fig. $4 \mathrm{~b}$ ). In addition, we found that Nox4 was upregulated by hypoxia at $1 \%(\mathrm{v} / \mathrm{v}) \mathrm{O}_{2}$ at both the mRNA and protein level (fig. 5). After incubation for $24 \mathrm{~h}$ in $1 \%(\mathrm{v} / \mathrm{v}) \mathrm{O}_{2}$, Nox 4 was significantly increased at the mRNA level (fig. 5a, b), and was gradually increased under hypoxic conditions up to $48 \mathrm{~h}$ at the protein level (fig. 5c, d).

\section{Role of Nox4 in Cell Proliferation}

We finally examined the effect of Nox4 on cell proliferation. Knockdown of Nox4 by RNAi significantly attenuated the proliferation of pericytes (fig. 6a, b), suggesting that Nox 4 participates in the proliferative process of human brain pericytes. 
Fig. 4. a Expression of the Ang II receptors in human brain pericytes. RT-PCR was performed using RNA $(1 \mu \mathrm{g})$ from human brain pericytes using specific primers for AT1R and AT2R. The PCR products were subjected to $1.2 \%(\mathrm{w} / \mathrm{v})$ agarose-gel electrophoresis, and stained with ethidium bromide. b Quantification of Nox4 and p $22^{\text {phox }}$ mRNA expression in brain pericytes in response to the addition of Ang II $(1 \mu \mathrm{M}, 24 \mathrm{~h})$. Real-time RT-PCR was performed using the LightCycler instrument (Roche). RNA (1 $\mu \mathrm{g})$ from human brain pericytes and specific primers for Nox4 and $\mathrm{p} 22^{\text {phox }}$ were used $(\mathrm{n}=4){ }^{*} \mathrm{p}<0.01$.

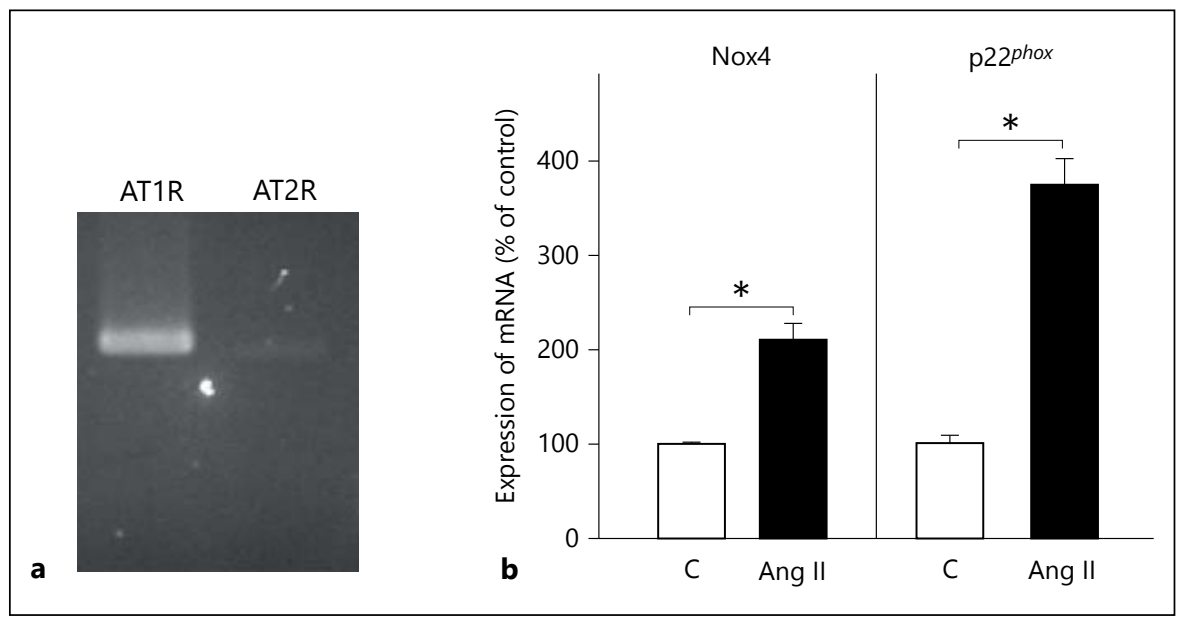

Fig. 5. Effect of hypoxia on Nox4 expression in human brain pericytes. Cultured pericytes were placed in a hypoxic chamber $\left(1 \%(\mathrm{v} / \mathrm{v}) \mathrm{O}_{2}\right)$. Twenty-four hours later, total RNA was isolated from these cells and subjected to RT-PCR (a). Quantification of Nox4 mRNA expression was performed using the LightCycler instrument (Roche) $(\mathrm{n}=4) .{ }^{*} \mathrm{p}<0.01$ (b). c, d Western blot analysis was also performed using total cell lysates of human brain pericytes subjected to hypoxic conditions $\left(1 \%(\mathrm{v} / \mathrm{v}) \mathrm{O}_{2}\right)$ for up to $48 \mathrm{~h}$. Gel image (c) and its quantitative analysis using imageJ (d) are shown. The average value of the density of bands corresponding to control cells $(0 \mathrm{~h})$ was set as $100 \%(\mathrm{n}=4){ }^{*} \mathrm{p}<0.01$ versus control $(0 \mathrm{~h})$.

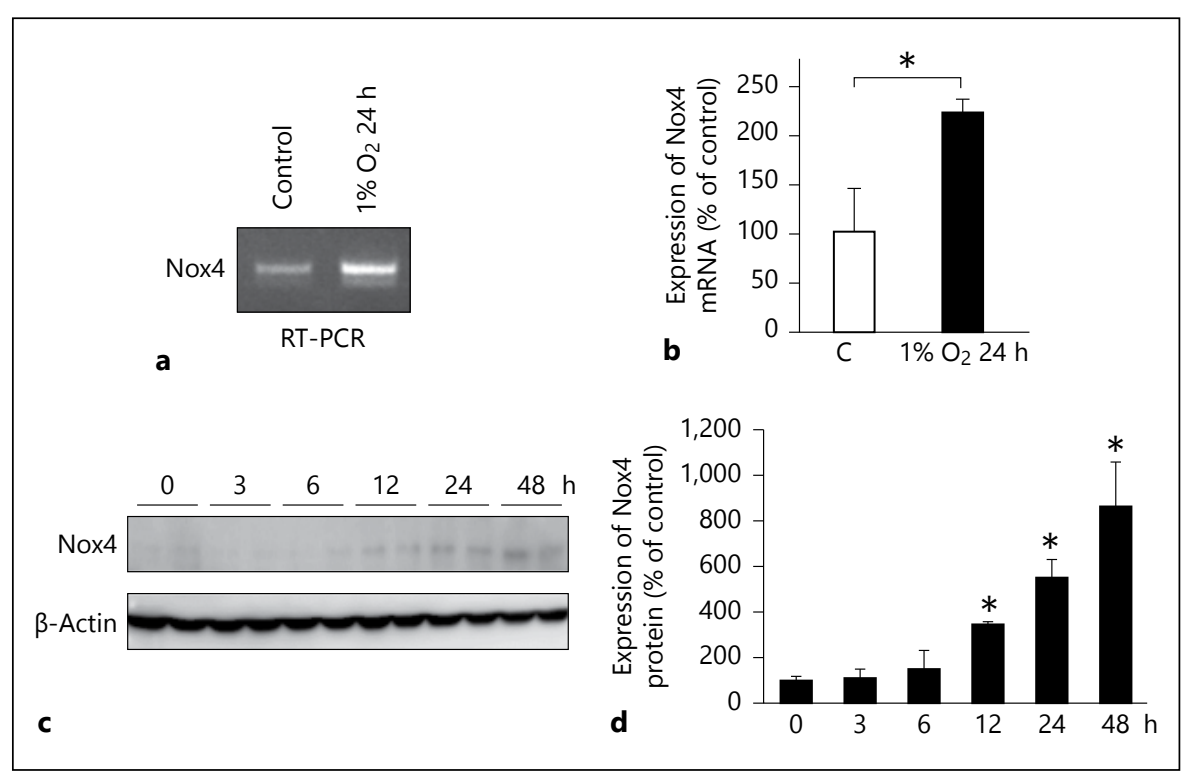

\section{Discussion}

In the present study, we demonstrated the following new findings. First, Nox4 is the predominant Nox protein in human brain pericytes. Second, Nox 4 is upregulated by Ang II and hypoxic stress, and third, Nox4 plays a role in proliferation of brain pericytes.

Previous studies have reported conflicting results regarding the expression of the Nox homologue in pericytes. It was reported that Nox1, but not Nox2/gp91 phox, was predominantly expressed in pericytes isolated from the rat adipose-tissue microvasculature [28]. Previous studies suggested that ROS are produced via NADPH oxidase in retinal pericytes, which are thought to be analo- gous to brain pericytes, in response to some stimulants $[29,30]$. It was shown that Nox2 (gp91 ${ }^{\text {phox }}$ ) was expressed and localized in the cytosol of bovine retinal pericytes; however, the expression of other Nox family members, including Nox4, in these cells was not investigated [30]. In the present study, we have clearly shown that Nox4 is expressed in human brain pericytes and that Nox4 participates in ROS production by the membrane fraction of these cells. However, we cannot conclusively determine if the same Nox isoform is responsible in various organs. We found that Nox5, which is present in humans but not in rodents, was faintly expressed in human brain pericytes; however, the present study suggests little role for Nox5 in human brain pericytes. 
Fig. 6. Effect of knockdown of Nox4 on the proliferation of pericytes. a Human brain pericytes were subjected to RNAi targeting Nox4. Forty-eight and $120 \mathrm{~h}$ after the transfection of siRNAs, cell number was counted. The average value of the cell number with control siRNA at $48 \mathrm{~h}$ was set as $100 \%$ $(\mathrm{n}=3) .{ }^{*} \mathrm{p}<0.01$. b Human brain pericytes were subjected to RNAi targeting Nox4 as above. The MTS assay was performed 48 and $120 \mathrm{~h}$ after the transfection of siRNAs. At each point of time, the average value of absorbance with control siRNA was set as $100 \%(\mathrm{n}=6){ }^{*} \mathrm{p}<0.05$.

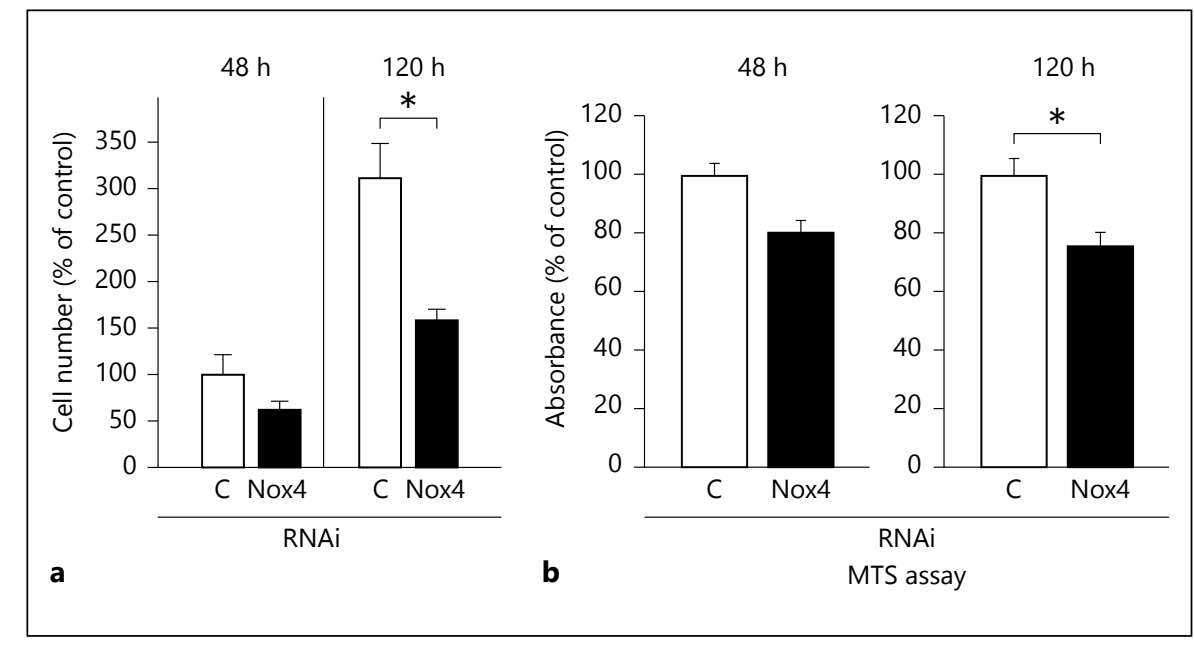

The membrane fraction of human brain pericytes produced superoxide following the addition of NADH as well as NADPH. However, the addition of the cytosolic fraction had no effect on superoxide production. These features are in accordance with the typical characteristics of Nox4 [25]. To explore whether Nox4 is a major source of ROS in brain pericytes, we further investigated the production of ROS by inactivating the enzyme by pharmacological agents as well as RNAi. Superoxide-producing activity was almost abolished by DPI, an inhibitor of Nox enzymes, whereas inhibitors of other potential sources of superoxide had no effect on the superoxide-producing activity. Silencing of Nox4 significantly reduced superoxide production. Taken together, in brain pericytes, Nox4 appears to be a major source of $\mathrm{NAD}(\mathrm{P}) \mathrm{H}$-dependent ROS production.

As one of the intrinsic regulators of Nox enzymes, Ang II occupies an important place in vascular pathophysiology. RAS plays a major role in vascular biology, particularly in relation to vascular disease. Ang II has diverse effects including vasoconstriction, oxidative stress, inflammation, endothelial dysfunction, impairment of neurovascular coupling, and vascular structure [31]. Blood vessels in the brain are constantly exposed to Ang II from both circulating and local sources. Ang II contributes to cerebrovascular diseases under conditions not only associated with hypertension but also with diabetes, arteriosclerosis, aging, and smoking. Moreover, RAS has been implicated in endothelial dysfunction and inflammation in cerebral microcirculation, through ROS production by Nox (Reviewed in [31]). In the present study, the Ang II receptors were expressed in brain pericytes, and the addition of Ang II significantly increased Nox4 and p2 $2^{\text {phox }}$ expression, suggesting that RAS works in pericytes through upregulation of Nox 4 and p22 phox. Thus, in brain pericytes, Nox4 may be one of the key factors in the pathophysiology of cerebrovascular diseases.

Pericytes have been proposed to play a pivotal role during brain ischemia and in the post-stroke period through mechanisms such as contraction of pericytes [32] and angiogenesis [2, 6]. Hypoxic stress has been shown to induce ROS production in the brain [33]. In the present study, we found that Nox4 expression in human brain pericytes was upregulated under hypoxic conditions $\left(1 \%(\mathrm{v} / \mathrm{v}) \mathrm{O}_{2}\right)$. In addition, in non-ischemic mouse brain, the expression of Nox 4 assessed by immunofluorescence was considerably low (data not shown), but was found clearly in the microvessels of peri-infarct area, suggesting that Nox4 may be upregulated under hypoxic condition also in vivo. These results raise the possibility that Nox 4 in pericytes, upregulated in ischemic brain, has a role in pathophysiological process of brain ischemia, such as ROS-induced pericyte contraction and dysfunction, leading to the impairment of microcirculation in ischemic stroke [34].

Nox4 was originally identified as an NADPH oxidase homologue highly expressed in the kidney [25, 35]. In addition to its strong expression in the kidney, Nox4 is also found in a variety of cells including vascular endothelial and smooth muscle cells $[10,20-22,36]$. Because Nox4 is also expressed in brain pericytes, the enzyme may have an important role in ROS production in the brain microcirculation. The function of Nox-derived ROS in the vascular system is complex and depends not only on the Nox isoform, but also on the cell type [17]. Overexpression of 
Nox1, one of the Nox family members, has been reported to increase the growth rate of NIH3T3 cells [37]. In the present study, Nox 4 was also involved in the proliferation of human brain pericytes. Proliferation of pericytes is an essential event in angiogenesis, in which these cells are capable of guiding sprouting processes by migrating ahead of endothelial cells or maturing the newly formed capillaries $[1,6,8]$. Taken together, superoxide production through Nox 4 in brain pericytes may be involved in angiogenesis in the brain by regulating the proliferation of cells.

We should note that the present study had some limitation regarding the methods. We used the cell-free chemiluminescence method for evaluation of ROS production by pericyte membrane under the condition of $\mathrm{NAD}(\mathrm{P}) \mathrm{H}$-dependency. Therefore, we should understand that the results were obtained under restricted conditions. It cannot be completely denied that ROS production by other pathways, such as electron leakage from the mitochondrial respiratory chain, nitric oxide synthases, and xanthine oxidases, have roles in human brain pericytes, in spite of using inhibitors of these pathways in this assay.
In conclusion, the present study revealed that Nox4 is a major source of superoxide production in human brain pericytes, and is regulated by Ang II and hypoxic stress in these cells. Nox4 in brain pericytes may have a central role in the pathophysiology of cerebrovascular diseases such as arteriosclerosis and ischemic stroke. Because pericytes are abundantly present in the brain vessels and are an important component of the neurovascular unit, Nox4 in brain pericytes may be a potential target for new therapies for cerebrovascular diseases.

\section{Acknowledgments}

We are grateful to Naoko Kasahara (Hisayama Research Institute for Lifestyle Diseases) and Chisato Tomita (Kyushu University) for their technical assistance.

This study was supported in part by a Grant-in-Aid for Scientific Research (C) (25461134) from the Ministry of Education, Culture, Sports, Science and Technology of Japan (to J.K.); the Takeda Science Foundation, Japan (to J.K.); and a Grant-in-Aid for Scientific Research (C) (26461145) from the Ministry of Education, Culture, Sports, Science and Technology of Japan (to T.A.).

\section{References}

-1 Shepro D, Morel NM: Pericyte physiology. FASEB J 1993; 7:1031-1038.

$\checkmark 2$ Armulik A, Abramsson A, Betsholtz C: Endothelial/pericyte interactions. Circ Res 2005 97:512-523.

3 Kutcher ME, Herman IM: The pericyte: cellular regulator of microvascular blood flow. Microvasc Res 2009;77:235-246.

4 Frank RN, Dutta S, Mancini MA: Pericyte coverage is greater in the retinal than in the cerebral capillaries of the rat. Invest Ophthalmol Vis Sci 1987;28:1086-1091.

5 Kamouchi M, Ago T, Kitazono T: Brain pericytes: emerging concepts and functional roles in brain homeostasis. Cell Mol Neurobiol 2011;31:175-193.

6 Kamouchi M, Ago T, Kuroda J, Kitazono T: The possible roles of brain pericytes in brain ischemia and stroke. Cell Mol Neurobiol 2012;32:159-165.

7 Fisher M: Pericyte signaling in the neurovascular unit. Stroke 2009;40:S13-S15.

8 Bergers G, Song S: The role of pericytes in blood-vessel formation and maintenance. Neuro Oncol 2005;7:452-464.

$>9$ Rucker HK, Wynder HJ, Thomas WE: Cellular mechanisms of CNS pericytes. Brain Res Bull 2000;51:363-369.

$>10$ Manea A: NADPH oxidase-derived reactive oxygen species: involvement in vascular physiology and pathology. Cell Tissue Res 2010; 342:325-339.
11 Harrison D, Griendling KK, Landmesser U, Hornig B, Drexler H: Role of oxidative stress in atherosclerosis. Am J Cardiol 2003;91:7A-11A.

12 Stocker R, Keaney JF Jr: Role of oxidative modifications in atherosclerosis. Physiol Rev 2004;84:1381-1478.

13 Ago T, Kuroda J, Kamouchi M, Sadoshima J, Kitazono T: Pathophysiological roles of NADPH oxidase/nox family proteins in the vascular system. Circ J 2011;75:1791-1800.

14 Brandes RP, Weissmann N, Schroder K NADPH oxidases in cardiovascular disease. Free Radic Biol Med 2010;49:687-706.

15 Rivera J, Sobey CG, Walduck AK, Drummond GR: Nox isoforms in vascular pathophysiology: insights from transgenic and knockout mouse models. Redox Rep 2010;15: 50-63.

16 Wolin MS, Ahmad M, Gupte SA: The sources of oxidative stress in the vessel wall. Kidney Int 2005;67:1659-1661.

-17 Bedard K, Krause KH: The NOX family of ROS-generating NADPH oxidases: physiology and pathophysiology. Physiol Rev 2007;87: 245-313.

18 Sumimoto H: Structure, regulation and evolution of Nox-family NADPH oxidases that produce reactive oxygen species. FEBS J 2008; 275:3249-3277.

19 Lambeth JD: Nox enzymes and the biology of reactive oxygen. Nat Rev Immunol 2004;4: 181-189.
20 Ago T, Kitazono T, Kuroda J, Kumai Y, Kamouchi M, Ooboshi H, Wakisaka M, Kawahara $\mathrm{T}$, Rokutan $\mathrm{K}$, Ibayashi $\mathrm{S}$, Iida $\mathrm{M}$ : $\mathrm{NAD}(\mathrm{P}) \mathrm{H}$ oxidases in rat basilar arterial endothelial cells. Stroke 2005;36:1040-1046.

21 Ago T, Kitazono T, Ooboshi H, Iyama T, Han YH, Takada J, Wakisaka M, Ibayashi S, Utsumi H, Iida M: Nox4 as the major catalytic component of an endothelial NAD(P)H oxidase. Circulation 2004;109:227-233.

22 Kuroda J, Nakagawa K, Yamasaki T, Nakamura K, Takeya R, Kuribayashi F, Imajoh-Ohmi S, Igarashi K, Shibata Y, Sueishi K, Sumimoto H: The superoxide-producing $\mathrm{NAD}(\mathrm{P}) \mathrm{H}$ oxidase Nox4 in the nucleus of human vascular endothelial cells. Genes Cells 2005;10:1139-1151.

-23 Arimura K, Ago T, Kamouchi M, Nakamura K, Ishitsuka K, Kuroda J, Sugimori H, Ooboshi H, Sasaki T, Kitazono T: PDGF receptor beta signaling in pericytes following ischemic brain injury. Curr Neurovasc Res 2012;9:1-9.

24 Tokami H, Ago T, Sugimori H, Kuroda J, Awano H, Suzuki K, Kiyohara Y, Kamouchi M, Kitazono T; REBIOS Investigators: RANTES has a potential to play a neuroprotective role in an autocrine/paracrine manner after ischemic stroke. Brain Res 2013;1517:122-132.

25 Shiose A, Kuroda J, Tsuruya K, Hirai M, Hirakata $\mathrm{H}$, Naito S, Hattori M, Sakaki Y, Sumimoto $\mathrm{H}$ : A novel superoxide-producing $\mathrm{NAD}(\mathrm{P}) \mathrm{H}$ oxidase in kidney. J Biol Chem 2001;276:1417-1423. 
26 Kuroda J, Ago T, Matsushima S, Zhai P, Schneider MD, Sadoshima J: NADPH oxidase 4 (Nox4) is a major source of oxidative stress in the failing heart. Proc Natl Acad Sci U S A 2010;107:15565-15570.

27 Elbashir SM, Harborth J, Lendeckel W, Yalcin A, Weber K, Tuschl T: Duplexes of 21-nucleotide RNAs mediate RNA interference in cultured mammalian cells. Nature 2001;411: 494-498.

28 Manea A, Raicu M, Simionescu M: Expression of functionally phagocyte-type NAD(P) $\mathrm{H}$ oxidase in pericytes: effect of angiotensin II and high glucose. Biol Cell 2005;97:723-734.

29 Kim J, Kim KM, Kim CS, Sohn E, Lee YM, Jo K, Kim JS: Puerarin inhibits the retinal pericyte apoptosis induced by advanced glycation end products in vitro and in vivo by inhibiting NADPH oxidase-related oxidative stress. Free Radic Biol Med 2012;53:357-365.
30 Mustapha NM, Tarr JM, Kohner EM, Chibber R: NADPH oxidase versus mitochondriaderived ROS in glucose-induced apoptosis of pericytes in early diabetic retinopathy. J Ophthalmol 2010;2010:746978.

31 Faraci FM: Protecting against vascular disease in brain. Am J Physiol Heart Circ Physiol 2011;300:H1566-H1582.

32 Dalkara T, Gursoy-Ozdemir Y, Yemisci M: Brain microvascular pericytes in health and disease. Acta Neuropathol 2011;122:1-9.

33 Allen CL, Bayraktutan U: Oxidative stress and its role in the pathogenesis of ischaemic stroke. Int J Stroke 2009;4:461-470.
34 Yemisci M, Gursoy-Ozdemir Y, Vural A, Can A, Topalkara K, Dalkara T: Pericyte contraction induced by oxidative-nitrative stress impairs capillary reflow despite successful opening of an occluded cerebral artery. Nat Med 2009;15:1031-1037.

35 Geiszt M, Kopp JB, Varnai P, Leto TL: Identification of renox, an $\mathrm{NAD}(\mathrm{P}) \mathrm{H}$ oxidase in kidney. Proc Natl Acad Sci U S A 2000;97: 8010-8014.

36 Lassegue B, Griendling KK: NADPH oxidases: functions and pathologies in the vasculature. Arterioscler Thromb Vasc Biol 2010;30: 653-661.

- 37 Suh YA, Arnold RS, Lassegue B, Shi J, Xu X, Sorescu D, Chung AB, Griendling KK, Lambeth JD: Cell transformation by the superoxide-generating oxidase Mox1. Nature 1999; 401:79-82. 